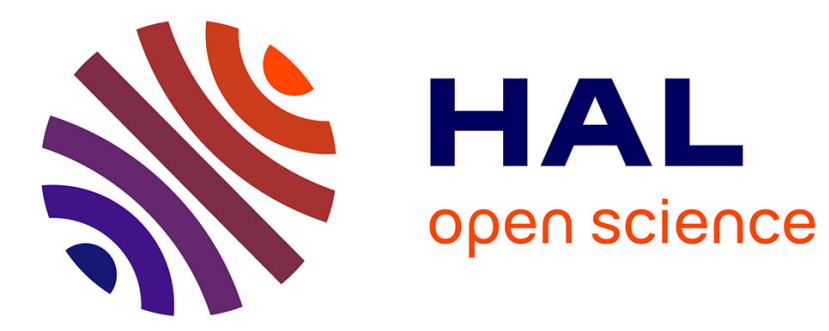

\title{
MATISSE, perspective of imaging in the mid-infrared at the VLTI
}

B. Lopez, P. Antonelli, S. Wolf, S. Lagarde, W. Jaffe, R. Navarro, U. Graser, R. Petrov, G. Weigelt, Y. Bresson, et al.

\section{- To cite this version:}

B. Lopez, P. Antonelli, S. Wolf, S. Lagarde, W. Jaffe, et al.. MATISSE, perspective of imaging in the mid-infrared at the VLTI. SPIE Astronomical Telescopes and Instrumentation, Jun 2008, Marseille, France. pp.1-10. hal-00335606

\section{HAL Id: hal-00335606 https://hal.science/hal-00335606}

Submitted on 30 Oct 2008

HAL is a multi-disciplinary open access archive for the deposit and dissemination of scientific research documents, whether they are published or not. The documents may come from teaching and research institutions in France or abroad, or from public or private research centers.
L'archive ouverte pluridisciplinaire HAL, est destinée au dépôt et à la diffusion de documents scientifiques de niveau recherche, publiés ou non, émanant des établissements d'enseignement et de recherche français ou étrangers, des laboratoires publics ou privés. 


\title{
MATISSE, perspective of imaging in the mid-infrared at the VLTI
}

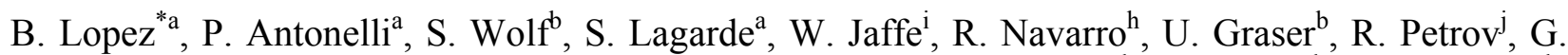
Weigelt $^{\mathrm{e}}$, Y. Bresson ${ }^{\mathrm{a}}$, K.H. Hofmann ${ }^{\mathrm{e}}$, U. Beckman ${ }^{\mathrm{e}}$, T. Henning ${ }^{\mathrm{b}}$, W. Laun ${ }^{\mathrm{b}}$, Ch. Leinert ${ }^{\mathrm{b}}$, S.Kraus $^{\mathrm{e}}$, S. Robbe-Dubois ${ }^{\mathrm{j}}$, F. Vakili $^{\mathrm{j}}$, A. Richichi ${ }^{\mathrm{p}}$, P. Abraham ${ }^{\mathrm{c}}$, J.-C. Augereau $^{\mathrm{d}}$, J. Behrend $^{\mathrm{e}}$, Ph. Berio $^{a}$, N. Berruyer ${ }^{a}$, O. Chesneau ${ }^{a}$, J.M. Clausse ${ }^{a}$, C. Connot ${ }^{e}$, K. Demyk ${ }^{f}$, W.C. Danchi ${ }^{g}$, M. Dugué $^{\mathrm{a}}$, G. Finger ${ }^{\mathrm{p}}$, S. Flament ${ }^{\mathrm{a}}$, A. Glazenborg ${ }^{\mathrm{h}}$, H. Hannenburg ${ }^{\mathrm{h}}$, M. Heininger ${ }^{\mathrm{e}}$, Y. Hugues ${ }^{\mathrm{a}}$, J. Hron $^{o}$, S. Jankov ${ }^{j}$, F. Kerschbaum ${ }^{o}$, G. Kroes ${ }^{h}$, H. Linz ${ }^{b}$, J.-L. Lizon ${ }^{p}$,Ph. Mathias ${ }^{a}$, R. Mathar ${ }^{i}$, A. Matter $^{\mathrm{a}}$, J.L. Menut ${ }^{\mathrm{a}}$, K. Meisenheimer ${ }^{\mathrm{b}}$, F. Millour ${ }^{\mathrm{j}}$, N. Nardetto ${ }^{\mathrm{e}}$, U. Neumann ${ }^{\mathrm{b}}$, E. Nussbaum ${ }^{\mathrm{e}}$, A. Niedzielski $^{\mathrm{k}}$, L. Mosoni ${ }^{\mathrm{b}, \mathrm{c}}$, J. Olofsson ${ }^{\mathrm{d}}$, Y. Rabbia ${ }^{\mathrm{a}}$, T. Ratzka $^{\mathrm{b}}$, F. Rigal ${ }^{\mathrm{h}}$, A. Roussel ${ }^{\mathrm{a}}$, D. Schertl ${ }^{\mathrm{e}}$, F.-X. Schmider, B. Stecklum ${ }^{1}$, E. Thiebaut ${ }^{\mathrm{m}}$, M. Vannier ${ }^{\mathrm{a}}$, B. Valat ${ }^{\mathrm{a}}$, K. Wagner ${ }^{\mathrm{b}}$, L.B.F.M. Waters ${ }^{\mathrm{n}}$.

a- Observatoire de la Côte d'Azur, Nice, France, b- Max Planck Institut für Astronomie, Heidelberg, Germany c- Konkoly Observatory Budapest, Hungary

d- Observatoire de Grenoble, Grenoble, France e- Max Planck Institut für Radioastronomie, Bonn, Germany, f- Université de Lille, Lille, France,

g- NASA Goddard Flight Center, Baltimore, USA, h- ASTRON, Dwingeloo, the Netherlands i- Leiden Observatory, the Netherlands j- Laboratoire Universitaire d'Astrophysique de Nice, France

k- Torun Centre for Astronomy, Torun, Poland 1- Thüringer Landessternwarte Tautenburg, Germany, m- Observatoire de Lyon, France, n- Astronomical Institute Amsterdam, the Netherlands, o- Vienna University, Austria, p- European Southern Observatory

\begin{abstract}
MATISSE is foreseen as a mid-infrared spectro-interferometer combining the beams of up to four UTs/ATs of the Very Large Telescope Interferometer (VLTI) of the European Southern Observatory. The related science case study demonstrates the enormous capability of a new generation mid-infrared beam combiner.

MATISSE will constitute an evolution of the two-beam interferometric instrument MIDI. MIDI is a very successful instrument which offers a perfect combination of spectral and angular resolution. New characteristics present in MATISSE will give access to the mapping and the distribution of the material (typically dust) in the circumstellar environments by using a wide mid-infrared band coverage extended to L, M and N spectral bands. The four beam combination of MATISSE provides an efficient UV-coverage : 6 visibility points are measured in one set and 4 closure phase relations which can provide aperture synthesis images in the mid-infrared spectral regime.
\end{abstract}

Keywords: Interferometry, Mid-Infrared Spectral Domain, VLTI.

*bruno.lopez@obs-nice.fr 


\section{INTRODUCTION}

The Very Large Telescope of the European Southern Observatory (ESO) recently opened a new era of astronomy for Europe. In particular, mid-infrared interferometry with the Mid-Infrared Interferometric Instrument for the VLTI, MIDI, operating since the end of the year 2002. It allows spatially resolved observations of reemission regions of hot dust in circumstellar disks, AGB stars, winds of hot stars, and the tori of AGNs with a resolution of 10-20 mas.

MIDI was proven to be very successful in interferometric and spectroscopic observations since it allows the comparison of the chemical composition of dust on very different spatial scales. However, the investigation of small-scale spatial structures in general, and the quantitative analysis of spectroscopic observations in particular, are strongly limited due to the small number of visibility points measured in a reasonable amount of time (over one or a few nights) and due to the lack of phase information.

Furthermore, the comparison between models and visibility points in the Fourier plane is currently the main strategy for the interpretation of MIDI observations.

The selection of more realistic models and the removal of possible ambiguities in the model fitting and resulting interpretations require a sufficient sampling of the uv-plane and the reconstruction of model-independent images.

Moreover, the classical analysis usually relies on two-dimensional models with rotation symmetry. This approach, which is only justified by large-scale -- if at all existent -- symmetries of the considered objects, is expected to be strongly misleading or simply wrong on the size scales investigated with MIDI.

In contrast, MATISSE will for the very first time allow image reconstruction of the small-scale regions traced with MIDI and thus finally allow an investigation of these structures based on an unprecedented level of constraints.

Our study underlines the interest for a higher spectral resolution and a large spectral coverage : L, M, and $\mathrm{N}$ bands. MATISSE will allow to perform interferometric spectroscopy with three different spectroscopic resolutions in the range of $\mathrm{R} \sim 30-1000$.

In particular, the following spectral resolutions are considered :

- $\mathrm{R}=30$ (amorphous dust features),

- $\quad \mathrm{R}=100--300$ (crystalline dust features, emission features of Polycylic Aromatic Hydrocarbons -- PAHs),

- $\quad$ and $\mathrm{R}=500--1000$ (e.g., molecular bands).

These spectral resolutions will allow a fundamental analysis of the composition of gases and dust grains in various environments. For example, MATISSE will be the first instrument ever able to probe the spatial distribution of CO ice in protoplanetary disks and thus provide a key in the understanding of the complex disk chemistry and planet formation.

In most astrophysical domains which require a multi-wavelength approach, MATISSE will be a perfect complement of forthcoming international high angular resolution facilities such as ALMA (the Atacama Large Millimeter Array). MATISSE covers the mid-infrared spectral domain, between the near-infrared domain, for which many interferometric facilities are developed, and ALMA which will operate at (sub) millimeter wavelengths. With the extended wavelength coverage from the $\mathrm{M}$ to the $\mathrm{N}$ band, MATISSE will not only allow to trace different spatial regions of the sources, but also different physical processes and thus provide insights into previously unexplored areas (such as the investigation of the distribution of volatiles in addition to that of the dust).

The mid-infrared spectral domain is very relevant for the study of the environment of various astrophysical sources. The MATISSE characteristics and expected sensitivity are given in the first part of the presentation.

Our science case studies show the wide field of applications of MATISSE. They will be illustrated in the second part of this presentation through the perspective of imaging the circumstellar environments/discs of young stellar objects. In a third part, a general view of the MATISSE modules is presented (the detailed design study being in progress). 


\section{INSTRUMENT CHARACTERISTICS}

The instrument characteristics derive from the goals defined in the 'Statement of Work and Technical Specifications for the Phase A Study of the MATISSE Instrument' (VLT-SPE-ESO-15860-0001), from the 'MATISSE Phase $\sim$ A Science Case' study (VLT-TRE-MAT-15860-4325), and, from the feasibility study and choices developed by the 'MATISSE Phase A System Design' (VLT-TRE-MAT-15860-4327).

MATISSE has the following characteristics :

- 4 Telescope beam combiner

$-3 \mathrm{~T}$ or $2 \mathrm{~T}$ configurations possible.

- Spectral Coverage

- Sensitivity optimized for $\mathrm{L}$ and $\mathrm{N}$ bands.

- other : M band.

- Simultaneous observations in $\mathrm{L}$ and $\mathrm{N}$ bands

- Spectral Resolution

- L $(\& M):$ Low $=30$, Medium $=300-500$, High $=750-1500$

$-\mathrm{N}:$ Low $=30, \mathrm{High}=300$

- Other

- Field rotation module

- 2D mode for acquisition

- Polarization filters for the $\mathrm{L}$ band

- Calibration devices (including Beam Commuter, Flat Field)

The performance goal in term of sensitivity is the following :

\begin{tabular}{|c|c|c|c|c|}
\hline \multicolumn{5}{|c|}{} \\
\cline { 2 - 5 } & N coherent flux limit & \multicolumn{2}{l|}{ L coherent flux limit } \\
\cline { 2 - 5 } & UT & AT & UT & AT \\
\hline Without fringe sensing or tracking & $0.4 \mathrm{Jy}$ & $8 \mathrm{Jy}$ & $0.03 \mathrm{Jy}$ & $0.6 \mathrm{Jy}$ \\
& $\mathrm{N}=5$ & $\mathrm{~N}=1.7$ & $\mathrm{~L}=9.9$ & $\mathrm{~L}=6.7$ \\
\hline With internal L band fringe sensing & $0.04 \mathrm{Jy}$ & $1 \mathrm{Jy}$ & $0.03 \mathrm{Jy}$ & $0.6 \mathrm{Jy}$ \\
& $\mathrm{N}=7.2$ & $\mathrm{~N}=4$ & $\mathrm{~L}=9.9$ & $\mathrm{~L}=6.7$ \\
\hline With external on-axis & $0.02 \mathrm{Jy}$ & $0.4 \mathrm{Jy}$ & $0.02 \mathrm{Jy}$ & $0.4 \mathrm{Jy}$ \\
K band fringe tracking & $\mathrm{N}=8.2$ & $\mathrm{~N}=5$ & $\mathrm{~L}=10.4$ & $\mathrm{~L}=7.1$ \\
\hline With external off-axis & $0.02 \mathrm{Jy}$ & $0.4 \mathrm{Jy}$ & $0.001 \mathrm{Jy}$ & $0.02 \mathrm{Jy}$ \\
K band fringe tracking & $\mathrm{N}=8.2$ & $\mathrm{~N}=5$ & $\mathrm{~L}=13.6$ & $\mathrm{~L}=10.4$ \\
\hline
\end{tabular}




\section{THE ADVANTAGE OF IMPROVING THE U-V PLANE COVERAGE FOR THE PERSPECTIVE OF IMAGING IN THE MID-INFRARED}

Observations in the different mid-infrared bands ( $, \mathrm{M}, \mathrm{N})$ will allow to trace regions with different characteristic temperatures, allowing to study the physical conditions and chemical environment in different spatial regions, such as in proto-planetary disks or stellar winds.

Furthermore, these observations will provide images with different spatial resolution, providing the basis for a comparison with lower-resolution images obtained at large telescopes with adaptive optics -- tracing the large-scale structure of the scientific targets -- in different wavelength regions (e.g., L/M band: NACO; N/Q band: VISIR).

Beside an individual analysis of measurements in different bands, a combination is thus expected to provide much stronger constraints on models of any of the potential science targets than $\mathrm{N}$ band observations alone. Depending on the individual band, unique spectral features (dust/gas) are accessible.

\section{THE SCIENTIFIC POTENTIAL OF THE SPECTROSCOPIC MODES}

MATISSE will allow to perform interferometric spectroscopy with three different spectroscopic resolutions. In particular, the following spectral resolutions are considered:

- $\quad$ Spectroscopic resolution $\mathrm{R}=30$ (amorphous dust features),

- $\quad \mathrm{R}=100--300$ (crystalline dust features, emission features of PAHs),

- $\quad$ and $\mathrm{R}=500-1000$ (molecular bands, selected atomic lines).

The potential of the different modes, which are focussed on different scientific aspects, are outlined in brief below.

Gas: The 3 to $13 \square \mathrm{m}$ spectral region is extremely rich in spectral diagnostics of gas covering a huge range in physical and chemical conditions. Strong permitted transitions of HI, HeI, and HeII probe ionized gas covering a wide range in density.

The MATISSE spectral region contains a large number of strong ro-vibrational bands of abundant molecules such as $\mathrm{CO}, \mathrm{OH}, \mathrm{H}_{2} \mathrm{O}, \mathrm{SiO}, \mathrm{C}_{2} \mathrm{H}_{2}, \mathrm{HCN}, \mathrm{CS}, \mathrm{SiC}$, that probe molecular gas near young and old stars, as well as in molecular clouds. The gas-phase diagnostics require spectral resolutions of the order of $10^{3}$ for optimal detection sensitivity.

Dust: The MATISSE spectral region contains the strongest resonances of abundant carbon-rich and oxygen-rich dust species. Oxygen-rich dust shows prominent bands at $9.7 \mu \mathrm{m}$ of amorphous silicates, and several bands of crystalline silicates, such as the forsterite $\mathrm{Mg}_{2} \mathrm{SiO}_{4}$ bands at 11.3 and $12.0 \mu \mathrm{m}$. In addition, several simple oxides have resonances,

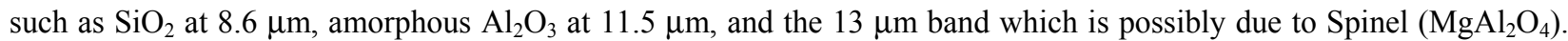
Carbon-rich dust has resonances of $\mathrm{SiC}$ at $11.3 \mu \mathrm{m}$, sometimes attributed to $\mathrm{TiC}$.

Furthermore, the famous UIR bands, now commonly attributed to PAHs have their strongest vibrational bands in the MATISSE spectral range (e.g. 3.3, 3.4, 7.7, 8.6, 11.3, $12.7 \mu \mathrm{m}$ ), due to $\mathrm{C}-\mathrm{H}$ and $\mathrm{C}-\mathrm{C}$ stretching and bending modes of these aromatic molecules. The $3.52 \mu \mathrm{m}$ band is believed to be due to nano-diamonds. The spectral resolution required to detect these dust species is of the order of 50 to 100, while studies of the mineralogy of the dust require a spectral resolution of $10^{3}$. Volatile dust or ice species have resonances somewhat displaced in wavelength compared to the gasphase molecules they form. The most prominent ice band is due to $\mathrm{H}_{2} \mathrm{O}$ and is near $3.1 \mu \mathrm{m}$.

The shape of the band is sensitive to the ice lattice structure, i.e. whether the ice is crystalline or amorphous. A second water ice band can be found near $12 \mu \mathrm{m}$. A second ice band accessible for MATISSE is CO at $4.7 \mu \mathrm{m}$.

MATISSE will be the first instrument ever to probe the spatial distribution of these ices, providing a key for understanding the complex chemistry of these disks. 


\section{SCIENCE CASES FOR MATISSE}

The following areas were identified as those where MATISSE will have a major impact by addressing qualitatively new questions (the scientific potential of MATISSE is outlined in the corresponding document, 'MATISSE Phase A Science Case' study (VLT-TRE-MAT-15860-4325, $1^{\text {st }}$ of June 2007) and in the 'Complement to the Science Case Document' (VLT-TRE-MAT-15860-4336, $15^{\text {th }}$ of September 2007) :

- Planet formation,

- Star formation (low and massive stars, multiple stars),

- Circumstellar disk evolution, ,

- Active Galactic Nuclei,

- Extrasolar planets,

- Solar System Minor Bodies,

- Dust and winds from evolved stars,

To illustrate the potential of MATISSE, we outline the example of planet formation studies in the following discussion.

\section{Example: Planet Formation}

Current star formation models predict the existence of circumstellar disks around young stellar objects as a natural consequence of the star formation process.

Given the importance and the strong interest in the astronomical community to study circumstellar disks in order to reveal the origin of planetary systems such as our own, circumstellar disks are key subject of observations by all available means. Unfortunately, the innermost region of disks with a radius of a few AU, where planet formation is expected to take place, can only be marginally investigated so far by the study of the spectral energy distribution, single visibility measurements (e.g. with MIDI or AMBER), high-resolution images obtained with the Hubble Space Telescope or by using adaptive optics.

This will remain the case until ALMA will be in operation with its longest baselines $(\sim 2012)$, unless one can provide high angular resolution images in the mid-infrared wavelength range.

In contrast to the typically ambiguous interpretations of visibilities as obtained with MIDI, MATISSE is aimed at answering pivotal questions about the inner regions of young circumstellar disks, such as:

\section{[1] What is the surface brightness profile in circumstellar disks around T Tauri HAeBe stars ?}

MATISSE will allow to consider that circumstellar disks which are not seen exactly face-on do show a brightness profile which cannot be described by iso-brightness contours, which are centered on the location of the central star, as assumed in the previous data analysis. It will permit to derive the radial temperature profile of the hot dust on the disk surface and the inner disk rim. This profile will provide information about the radial and vertical structure of the disk, and thus on the physical conditions in the potentially planet-forming regions of circumstellar disks.

\section{[2] Does the brightness profile show evidence for dust grain growth and sedimentation?}

MATISSE will allow to provide strong observational constraints for the region, where dust grain growth is presumably fastest.

As simulations of dust settling in circumstellar disks show, the disk flaring and thus the ability to absorb stellar radiation even at large distances from the star depends on the grain size distribution remaining in the upper disk layers -- an effect that can also be investigated with MATISSE (Dullemond and Dominik 2004). 


\section{[3] Is the inner disk structure modified by early stages of planet formation ?}

The inner region of circumstellar disks is expected to show large-scale (sub-AU - AU sized) density fluctuations / inhomogenities. Locally increased densities are expected to show up as local brightness variation (due to increased absorption/ shadowing effects) in the mid-infrared images (see Figure 1).

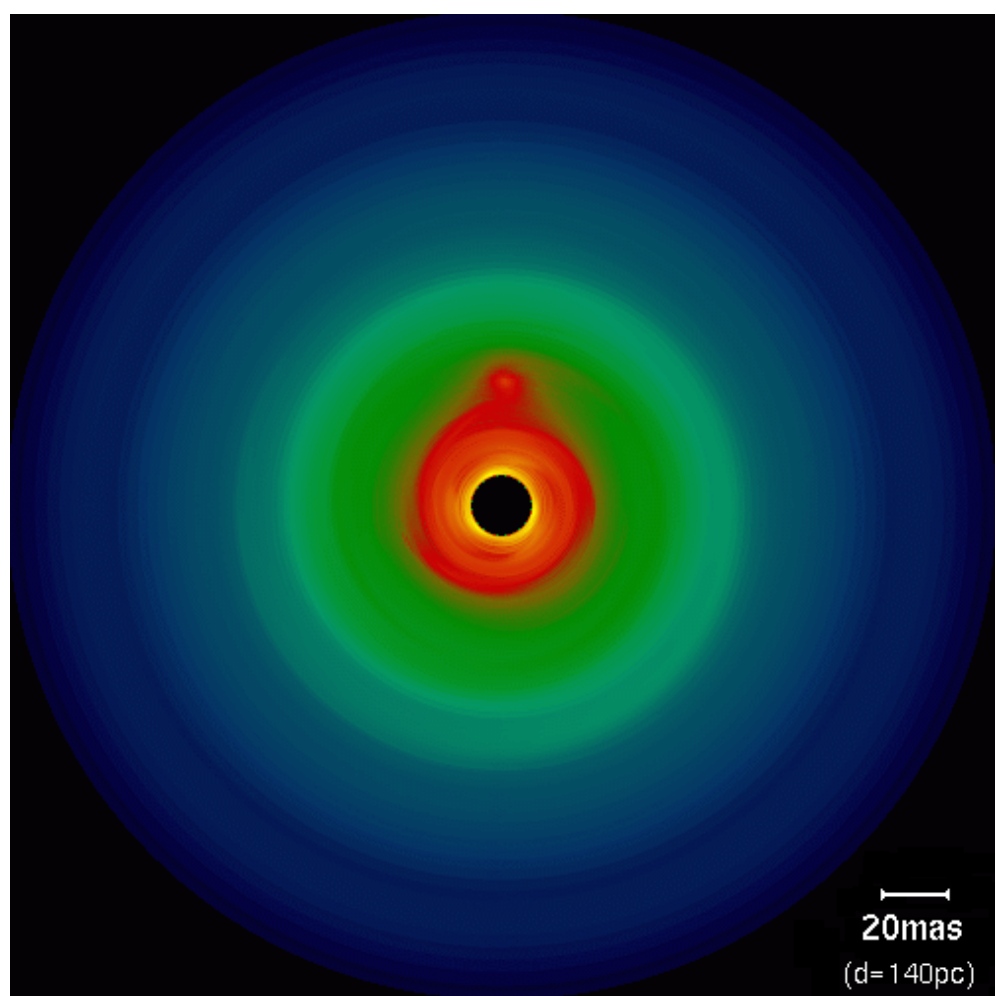

Fig. 1: Simulated $10 \mu \mathrm{m}$ images of the inner region of a circumstellar T Tauri disk, with an embedded Jupiter-mass planet at a distance of 5.2 AU from the central star (Wolf and Klahr, in prep.). The hot region around the planet above the center of the disk is indicated as bright areas in these reemission images. Assuming a distance of $140 \mathrm{pc}$, a 20 mas scale is indicated.

\section{[4] Is there indirect or even direct evidence for the presence of planets ?}

Once planets have been formed, they may significantly alter the surface density profile of the disk and thus cause signatures in the disk that are much easier to find than the planets themselves.

The appearance and type of these signatures depend on the mass and orbit of the planet, but even more on the evolutionary stage of the circumstellar disk. MATISSE would for example allow to observe a gap, which is expected to be created along the orbit of a young giant planet within the inner few AU of a young gas-dominated disks (Wolf 2006).

\section{A BRIEF VIEW OF THE DESIGN}

MATISSE is composed of several sub-systems. The first one is called "Warm optics". It represents the instrumental part between the VLTI feeding optics and the cryostat windows. Located on an optical table of $1.5 \times 2.1 \mathrm{~m}$ size, it is composed by 5 modules : the field rotation compensator, the beam commuting device, the spectral separator, the anamorphic optics and the delay lines. 
The field rotation compensator optimizes the field orientation in regards to the slits of the spectrograph and compensates the field and the polarization rotation existing in the focal laboratory.

The beam commutating device is used as a calibration tool for instrumental effects (including chromatic and detector effect) by inverting them thanks to beam commutations.

A spectral separation between the $\mathrm{L}$ (and $\mathrm{M}$ ) band and the $\mathrm{N}$ band is necessary because the beam combination modes, the materials of optics and the detectors are different. This separation is made by a set of 4 dichroïcs.

The anamorphic optics is used to make an image magnification in the spatial direction in order to optimize the fringe sampling.

The delay lines (one set per spectral band) transfer the pupil at the cold pupil mask level of the spectrograph and are used for fine OPD adjustment.

The cold optics represents the MATISSE part between the cryostat windows and the detector. It is made up of 2 separate cryostats (one for each spectral band). The functionalities of each cryostat are similar. They are composed by several elements:

The shutters select the chosen beams (permitting observations with 2, 3 or 4 telescopes).

The pupil masks reduce the thermal background (in particular the thermal background emitted by the optical mounts outside the cryostat) and avoid parasitic light.

Focusing mirrors produce an intermediate image to insert pinholes or slits used as spatial filter. After the spatial filter, collimating mirrors collimates the beams.

The pair separator composed by beam splitters and mirrors module separates the 4 beams in 6 pairs of beam. By combining different positions of shutters and beam splitter, several configurations of observation can be obtained: 4 telescopes without specific photometric images, 3 telescopes with specific photometric images and 2 telescopes with or without photometric images.

The $\pi$ phase shifter separates in two parts each pair of beam and produces a $\pi$ phase between these two outputs.

In case of observations with 4 telescopes, 12 images are produced ( 2 images per pair and 6 pairs of beam). 12 tilted flat mirrors introduce a slight angle between the beams in order to position all these images at different locations on the detector.

Prism and grisms can be chosen to obtain the desired spectral resolution.

The camera optics focalize the beam on the detectors and is the beam combiner (multi-axial combination) of the instrument.

The Figure 2 gives the general implementation of the MATISSE instrument. The warm optical table in the centre of the figure has a size of about $1.2 \times 2.4 \mathrm{~m}$ (TBC). The two cryostats are located symmetrically on the two sides of the warm optical bench. 


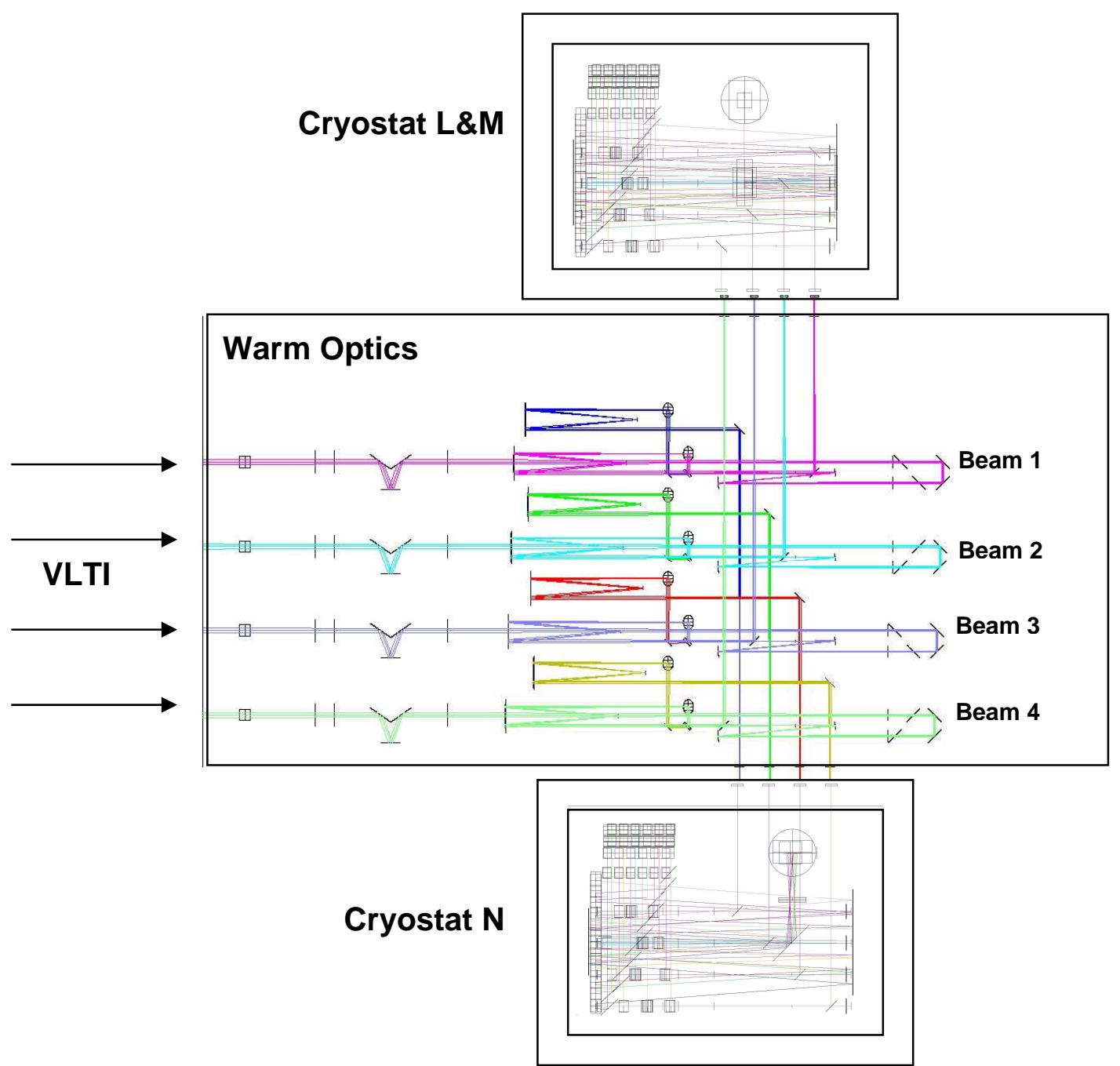

Fig. 2: General top view of the optical design of MATISSE

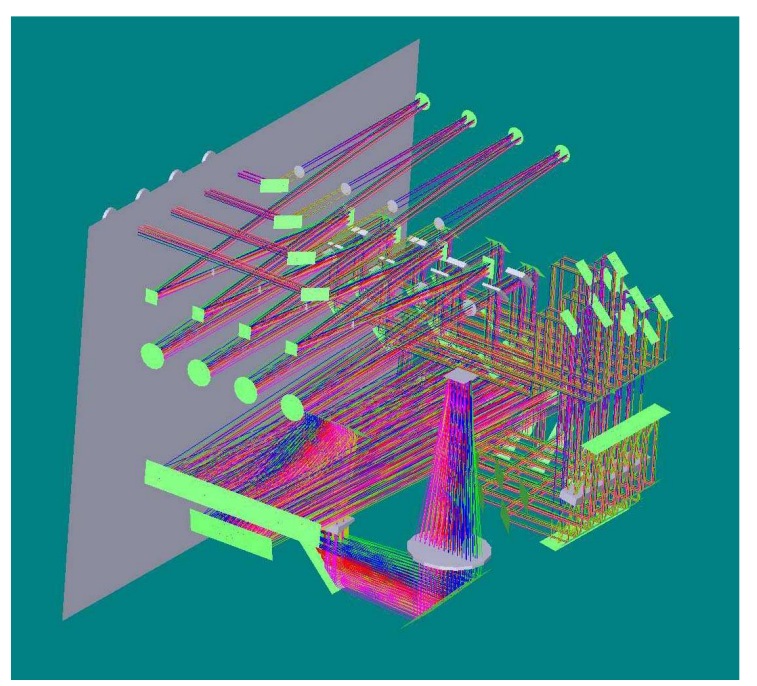

Fig. 3: General view of the cold optics of MATISSE 
The Figure 3 is a 3D view of the cryostat. On the left side behind the "grey wall" we can identify the four input windows. Then the beams are deviated by four mirrors towards four couples of parabolic mirrors (image plane, spatial filtering). After the spatial filtering module, in the centre of the figure we can identify the pair creator module followed by the OPD module (centre - right). At the bottom right we can see the $0-\pi$ module followed by the tip/tilt adjustment mirrors for re-positioning the pairs onto the detector. Several flat mirrors are placed before the camera optics and the detector.

\section{CONCLUSION}

There exist only two other $10 \mu \mathrm{m}$ ground based long-baseline interferometers (ISI 3 and Keck I) and two projects presently under study for mid-infrared space interferometry (Darwin and TPF I).

The Infrared Stellar Interferometer ISI 3, equipped with three $1.6 \mathrm{~m}$ telescopes, allows imaging and operates in heterodyne mode at a moderate sensitivity level, related to the very narrow band used.

The Keck Interferometer, on the other hand, has two large apertures on fixed baselines. Its sensitivity is equivalent to that of the VLTI. However, its imaging capability is only based on the supersynthesis effect which allows the exploration of a limited range of spatial frequencies in the Fourier plane.

Darwin and TPF I (Terrestrial Planet Finder Interferometer) are space projects, particularly focused on detecting earthlike extrasolar planets and the search for bio-signatures in their atmospheres.

MATISSE is a unique instrument (see Figure 4). It offers new scientific breakthroughs and will contribute to the evolution of mid-infrared interferometry.

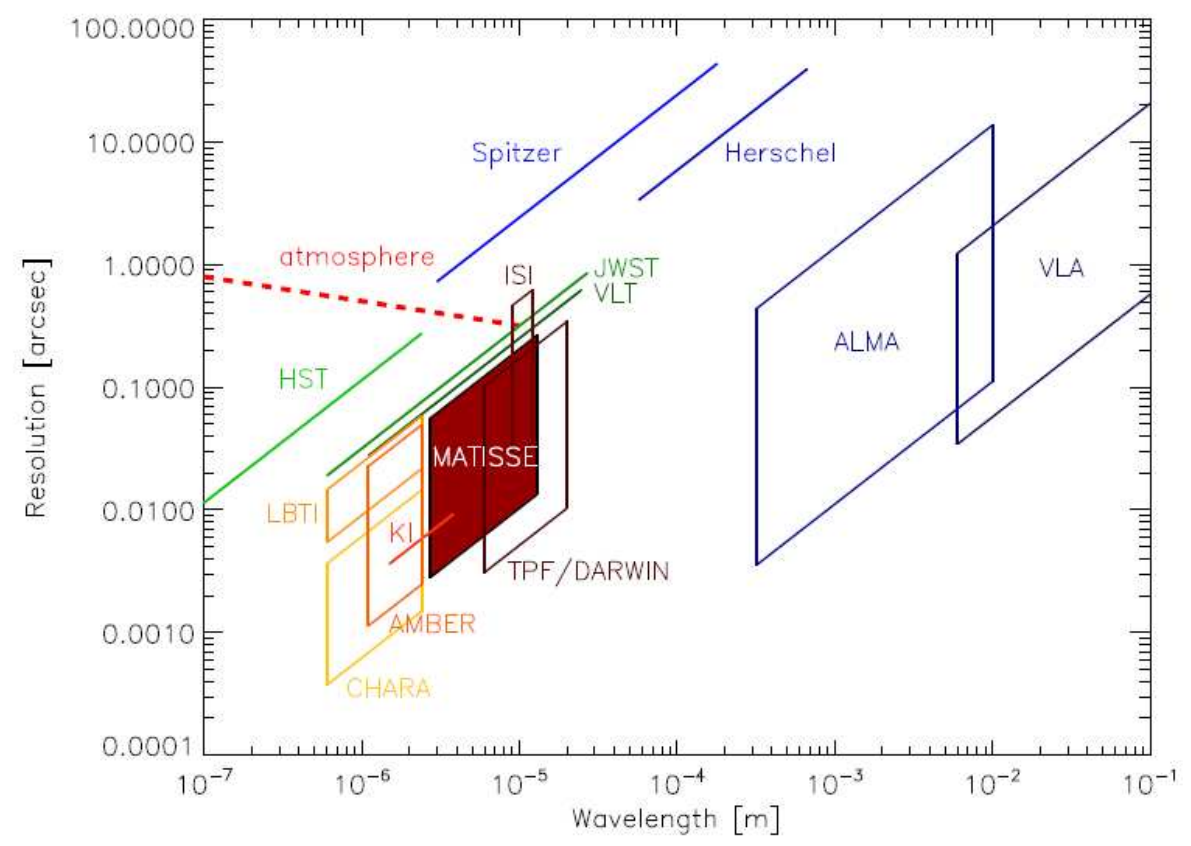

Fig. 4: Uniqueness of MATISSE at theVLTI represented in a Spatial Resolution versus Wavelength diagram. 


\section{REFERENCES}

Dullemond C.P. and Dominik C., 2004, A\&A 421, 1075.

Lagarde et al., 2006, these Proceedings.

'MATISSE Phase A Science Case' study, VLT-TRE-MAT-15860-4325, $1^{\text {st }}$ of June 2007, Wolf S., Lopez B., et al..

Wolf S., 2006, in 'Direct Imaging of Exoplanets: Science \& Techniques', IAU Colloquium \#200, p139.

'MATISSE : Concept Analysis', Lagarde S. et al., this Proceeding.

'Aperture-Synthesis Imaging with the mid-Infrared Instrument MATISSE', Hofmann K.-H. et al., this Proceeding.

'MATISSE Phase A System Design', VLT-TRE-MAT-15860-4327, $1^{\text {st }}$ of June 2007, Lagarde S. et al..

'Complement to the Science Case Document', VLT-TRE-MAT-15860-4336, $15^{\text {th }}$ of September 2007, Lopez B. et al.. 